\title{
EMPIRISCHE WIRTSCHAFTS- UND SOZIALFORSCHUNG
}

\author{
Herausgegeben von \\ Prof. Dr. Gerold Blümle \\ Prof. Dr. Hans-Josef Brink \\ Prof. Dr. Siegfried Hauser
}

Band 14 


\title{
Datenvisualisierung
}

\author{
Neuere Entwicklungen \\ der explorativen graphischen Datenanalyse \\ mittels metrischer Skalierungsverfahren
}

\section{Ariane Kristof}

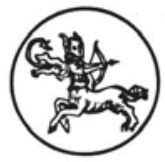

Centaurus Verlag \& Media UG 1995 
Die Deutsche Bibliothek - CIP-Einheitsaufnahme

Kristof, Ariane:

Datenvisualisierung : neuere Entwicklungen der explorativen graphischen Datenanalyse

mittels metrischer Skalierungsverfahren / Ariane Kristof. Pfaffenweiler : Centaurus-Verl.-Ges., 1995

(Empirische Wirtschafts- und Sozialforschung ; 14)

Zugl.: Freiburg (Breisgau), Univ., Diss., 1993

ISBN 978-3-89085-972-9 ISBN 978-3-86226-326-4 (eBook)

DOI 10.1007/978-3-86226-326-4

NE: GT

\section{ISSN 0935-0365}

Alle Rechte, insbesondere das Recht der Vervielfältigung und Verbreitung sowie der Übersetzung, vorbehalten. Kein Teil des Werkes darf in irgendeiner Form (durch Fotokopie, Mikrofilm oder ein anderes Verfahren) ohne schriftliche Genehmigung des Verlages reproduziert oder unter Verwendung elektronischer Systeme verarbeitet, vervielfältigt oder verbreitet werden.

(c) CENTAURUS-Verlagsgesellschaft mit beschränkter Haftung, Pfaffenweiler 1995

Satz: Vorlage der Autorin 


\section{Inhaltsverzeichnis}

Verzeichnis der Tabellen

Seite

Verzeichnis der Abbildungen

iv

Abkürzungsverzeichnis

iv

I. Einführung in das Problemgebiet

II. Warum Daten graphisch aufbereiten?

II.1. Zur Geschichte der graphischen Aufbereitung von Daten 8

II.2. Die verschiedenen Funktionen moderner graphischer Datenanalyse 13

II.3. Zur Philosophie der explorativen Datenanalyse (EDA) 19

II.4. Zur Geschichte und Philosophie der Korrespondenzanalyse 27

II.5. Graphische Perzeptionstheorie 34

III. Ausgewăhlte Methoden zur Abbildung multivariater Daten 41

III.1 Tukeys Stamm-und-Blatt-Diagramm 41

III.2. Polygone $r$

$\begin{array}{ll}\text { III.3. Chernoff-Gesichter } & 43\end{array}$

III.4. Andrews-Kurven $\quad 46$

$\begin{array}{ll}\text { III.5. Vergleich mit der Korrespondenzanalyse } & 48\end{array}$

IV. Die Geometrie der Hauptkomponentenanalyse 50

$\begin{array}{ll}\text { IV.1. Grundlegende Bemerkungen } & 50\end{array}$

IV.2. Definition einer Punktwolke im einem

IV.3. Definition einer Metrik für den Datenraum 52

IV.4. Einpassung der Punktwolke in einen niedrig-

$\begin{array}{ll}\text { IV.5. Weitergehende Bemerkungen } & 61\end{array}$ 
V.1. Einführende Bemerkungen

V.2. Definition einer Punktwolke in einem multidimensionalen Vektorraum

v.3. Definition der Metrik für den Vektorraum

V.4. Einpassung der Punktwolke in einen niedrigdimensionierten Unterraum

V.5. Zur Darstellung der Korrespondenzanalyse in der Literatur: Die SVD

VI. Zur Anwendung der Korrespondenzanalyse in den Wirtschaftswissenschaften

VI.1. Grundlegende Oberlegungen zum Einsatz und zur Interpretation einer Korrespondenzanalyse

VI.2. Ausgewăhlte Anwendungsbeispiele der Korrespondenzanalyse

VI.2.1. Sitzverteilung im Deutschen Bundestag

VI.2.2. Portfolioanalysen

VI.2.3. Preisstrukturen innerhalb der $E G$

VI.3. Weiterentwicklungen der Korrespondenzanalyse

VI.3.1. Zusätzliche Profile und Vergleichbarkeit von Zeilen- und Spaltenkategorien

VI. 3.2. Multiple Korrespondenzanalyse

VI.4. Zusammenfassung und Versuch einer epistemologischen Einordnung der Korrespondenzanalyse 


\section{Verzeichnis der Tabellen}

Seite

$\begin{array}{llr}\text { Tabelle I.1: } & \text { Sitzverteilung im Deutschen Bundestag } & 4 \\ \text { Tabelle VI.1: } & \text { Sitzverteilung im Deutschen Bundestag } & 88 \\ \text { Tabelle VI.2: } & \text { Zeilenprofile aus Tabelle VI.1 } & 91 \\ \text { Tabelle VI.3: } & \text { Zeilenbeiträge zu Tabelle VI.1 } & 92 \\ \text { Tabelle VI.4: } & \text { The perfect portfolio? } & 95 \\ \text { Tabelle VI.5: } & \text { Wähungsanteile von Bond-Portfolios } & 96 \\ \text { Tabelle VI.6: } & \text { Zeilen- und Spaltenprofile zu Tabelle VI.5 } & 97 \\ \text { Tabelle VI.7: } & \text { A shopper's guide to the single market } & 100 \\ \text { Tabelle VI.8: } & \text { Ausgewählte Konsumgüterpreise } & 101 \\ \text { Tabelle VI.9: } & \text { Zeilenprofile aus Tabelle VI.8 } & 102 \\ \text { Tabelle VI.10: } & \text { Anpassungskennzahlen zu Abb. VI.4 } \\ \text { Tabelle VI.11: } & \text { Sitzverteilung im Deutschen Bundestag } & 105\end{array}$

\section{Verzeichnis der Abbildungen}

Abb. I.1:

Graphischer Output einer Korrespondenzanalyse

Abb. II. 1:

Originalbeispiel von M.O. Lorenz (Lorenzkurve) 11

Abb. II. 2 :

Schematisches Diagramm einer Clusteranalyse 25

Abb. III. 1:

OECD-Polygone

Abb. III. 2 :

Originalbeispiel für Chernoff-Gesichter

Abb. III. 3 :

Die Abb. III.2 zugehörige Tabellenfassung

Abb. III. 4:

Originalbeispiel für Andrews-Kurven

Abb. IV. 1:

Umorientierung einer Menge Punkte

Abb. IV. 2 :

Unterraumprojektion

Abb. VI.1:

KA-Schaubild der Zeilenprofile von Tabelle VI.1 89

Abb. VI. 2:

KA-Diagramm der entsprechenden Spaltenprofile

Abb. VI. 3: Symmetrisches KA-Diagramm von Tabelle VI.5

Abb. VI. 4 :

KA-Diagramm der europäischen Hauptstådte

Abb. VI. 5:

Projektion zusätzlicher Profile 


\section{Abkürzungsverzeichnis}

$\begin{array}{ll}\text { CLA } & \text { Clusteranalyse } \\ \text { DA } & \text { Diskriminanzanalyse } \\ \text { EDA } & \text { exploratory data analysis/explorative Datenanalyse } \\ \text { HKA } & \text { Hauptkomponentenanalyse } \\ \text { RA } & \text { Korrespondenzanalyse } \\ \text { MKA } & \text { multiple Korrespondenzanalyse } \\ \text { SVD } & \text { singular value decomposition }\end{array}$

